\title{
MOLECULES OF WATER IN SOLIDS
}

\begin{abstract}
A CONFERENCE was held in Paris during June A 24-26 under the auspices of the Centre National de la Recherche Scientifique, supported by the Rockefeller Foundation. The conference was the fifty-third in the series of discussions organized by the Centre National, and, as in previous conferences, it was limited to about twenty specialists. The full title of the discussion was "The Study of Molecules of Water in Solids by means of Electro-Magnetic Waves (from X-Rays to Radiofrequencies)". All the meetings except the last were held in the Institut de Chimie-Physique, of the Sorbonne, Paris, and the last session was held in one of the laboratories at the Chateau Gif. The following topics were discussed : (a) crystal structure and the physical properties of hydrates; (b) dielectric constants and dielectric absorption as a function of frequency and temperature; (c) infra-red spectra due to $\mathrm{H}_{2} \mathrm{O}$ and $\mathrm{OH}$ groups ; (d) Raman spectra; (e) magnetic resonance.

The papers presented at the conference showed that considerable advances are being made in the study of the water molecule in crystalline hydrates. Among the new techniques should be mentioned nuclear resonance and Raman spectra. The interaction of the protons within each water molecule makes it possible to determine, with a precision of a few degrees, the direction of the line joining the protons. Magnetic resonance measurements can also frequently afford a reasonably accurate measure of the distance apart of the protons. However, no information can at present be gained by this method concerning the relative positions of the oxygen and hydrogen atoms within any one molecule. Even with this limitation, the new method is complementary to the methods of $\mathrm{X}$-ray crystallography, which are primarily useful in fixing the position of the oxygen atoms. The frequencies of the Raman spectra are determined by the modes of vibration of the water molecule and the polarization of the Raman lines by the orientation of the molecule. When the mode of vibration can be identified by the frequency and when the crystal structure is not too complicated, it is possible to determine the direction of the $\mathrm{OH}$ bonds. This, too, is information complementary to that obtainable by X-rays. A present limitation on both these methods is the large size of crystal
\end{abstract} required.

The advances made using the older methods are due to the greater detail in the information available. For example, ninety-six crystal structures of hydrates have been established, and it has therefore become possible to devise a classification of hydrate structures based on the clustering of the water molecules into island-like, fibre-like, leaf-like or cage-like arrangements. To cover these categories the words neso-, ino-, phyllo-, and tecto-hydrates have been adopted from the earlier classification of the silicates. This classification brings out the variation in the way the water is included in the structure-a variation extending from practically free water, through hydroxyl and/or hydrogen bonds, to the oxonium ion.

The dielectric constant and dielectric absorption as functions of frequency and temperature have been extensively studied, though it must be said that the results do not lead to any fundamentally now information. Relaxation time and energy of activation can often be determined; but the dynamics of the water molecule in crystalline hydrates are still beyond adequate mathematical treatment.

Numerous infra-red spectra, both of powders and of single crystals, in polarized infra-red radiation have been studied. Bands of absorption or selective reflexion can be identified in many hydrates with the type of binding of the water molecule present. The influence of the surrounding ions can be traced in the changes in frequency and band-width. Like the studies on dielectric properties, the infra-red properties are often of considerable industrial importance, in determining the amount and kind of binding of the water present.

Lastly, enough information has become available to make possible some correlation of the crystal structure of hydrates with their coefficients of thermal expansion, dielectricity, piezoelectricity, elasticity and hardness.

It is intended to publish the papers presented to this conference in a fortheoming number of the Journal de Chimie Physique.

The following is a list of the authors and the titles of their contributions :

$X$-rays and physical properties. J. D. Bernal (London), "The role of water in crystalline substances"; P. G. Owston (London), "The position of the hydrogen atoms in ice"; W. A. Wooster (Cambridge), "The influence of molecules of water on the physical properties of crystalline hydrates".

Dielectric constants. R. Freymann (Rennes), "Absorption of free and bound water in Hertzian spectra"; "Orientation of molecules and lattice defects"; E. Bauer and M. Magat (Paris); "Dispersion of the dielectric constant in crystal hydrates"; J. B. Hasted (London), "Study of water molecules at radio frequencies"; A. Steinemann (Zurich), "Dielectric constant of ice"; J. Chapelle and A. Galy (Nancy), "Dielectric constants of some crystal hydrates"; P. Abadie, R. Charbonniere, A. Gidel, P. Girard and A. Guilbot (Paris), "Study by absorption spectra at radio frequencies of water in the crystallization of maltose and glucose and study of the states of water of sorption in 'amidon'".

Infra-red spectroscopy. J. Lecomte (Paris), "The study of water in solids by infra-red spectra"; $R$. Mecke (Freiburg-im-Breisgau), "Detection of $\mathrm{OH}$ in crystals by infra-red spectroscopy"; D. E. Bethell and N. Sheppard (Cambridge), "Infra-red spectra of nitric acid monohydrate"; A. M. Vergnoux (Montpellier), "Infra-red studies of the $\mathrm{OH}$ bands in crystals".

Raman spectra. J-P. Mathieu (Paris), "The Raman effect in hydrated crystals"; L. Couture-Mathieu (Paris), "The Raman effect in gypsum"; R. Lafont and Ch. Bouhet (Montpellier), "The water-bands in single crystals of magnesium and zinc sulphate heptahydrates"; C. Vassas-Dubuisson (Paris), "The Raman effect in a single crystal of lithium sulphate monohydrate"; J. Chapelle and A. Galy (Nancy), "Attempt at orientation of the molecules of water in hydrated crystals by means of the Raman effect"; J. Chapelle, G. Champier and C. Delain (Nancy), "Interpretation of the Raman spectra due to water molecules in Rochelle Salt".

Nuclear magnetic resonance. G. E. Pake (Washington University, Miss.), "Application of nuclear magnetic resonance to the study of the structures of 
crystals containing molecules of water"; M. Soutif and Y. Ayant (Grenoble), "Study of the water molecule in lithium sulphate monohydrate"; N. J. Poulis and G. E. G. Hardeman (Leyden), "Position of protons in $\mathrm{CuCl}_{2} \cdot 2 \mathrm{H}_{2} \mathrm{O}$ determined by the method of nuclear magnetic resonance"; W. Gordy (U.S.A.), "The spectra of water vapour in the millimetric range". W. A. Wooster

\section{INTERNATIONAL ELECTRO-ACOUSTICS CONGRESS}

A $\mathrm{N}$ "International Electro-acoustics Congress" was $A$ held in Holland during June 16-24. The congress, which was organized by a Dutch Committee, presided over by Prof. C. W. Kosten, was the first venture sponsored by the newly formed International Committee of Acoustics (I.C.A.), an offshoot of Unesco. It was held in the main at the Technische Hoogeschool, Delft, and was attended by more than three hundred people, representing many countries.

After addresses by the president of the Congress, H. E. the Minister of Education and Prof. R. H. Bolt, president of the International Committee of Acoustics, the Congress settled down to hear general reviews of progress in the seven sections into which the subject had been divided. These are summarized below.

Section I: Sound Recording. R. Vermeulen described improvements which have been made in disk and tape recording. He pointed out the need for defining standards in reproduction apparatus. Frequency response is not the only criterion; the psychological attitude of the hearer and the auditorium in which he listens play a large part in appreciation. Advances in stereophony were pointed out and-later in the Philips works-a two-channel demonstration was given to show that the illusion of space in such a system is still imperfect.

Section II. Public Address Systems. Erwin Meyer pointed out that the Ioud-speaker remains the weakest link in this type of transduction, which has become so important in modern life. Usually it is more important, at least in speech, to get the high frequency amplified than the bass. Different types of microphones and arrays of loud-speakers were described, and the advantages of delay pick-ups as used in St. Paul's, London, for large auditoria was explained.

Section III : Measurements. L. Beranek emphasized the need for measuring devices which include the listener as part of the system. This science of psycho-acoustics is still in its infancy; but many acoustic measurements are valueless without it. On the purely physical side, the most important developments are in high-frequency microphones for use in a wide range of conditions, in the measurement of acoustic and mechanical impedance.

Section IV: Audiometers. P. Chavasse also pointed out the need for listener research in this field, which should cover not only psychological reaction but also measurements of a more physical character like the impedance of the external ear, diffraction of sound by the head, artificial ears, and establishment of a norm of hearing for all countries.

Section V: Ultrasonics. G. Bradfield described recent advances in transducer design for this range, now going up to $3,000 \mathrm{Mc}$./s., particularly those involving barium titanate and the ferrites. Trans- ducers for pulse excitation using Rochelle salt or long nickel tubes with the distant end immersed in grease have also been used in delay lines, while for ultrasonic cutting tools, 'Permendur' is used. The characteristics of many of these were discussed through their equivalent circuits or Argand diagrams. More study is needed of the launching of waves in solids, where the energy tends to be transferred to and from transverse and longitudinal waves, complicating the process.

Section VI : Musical Acoustics. E. G. Richardson described the benefits which this branch has derived from advances in electrical tone synthesis. Recent research has shown the importance of a knowledge of the formants (varying timbre over the scale) and transients of musical instruments in differentiating them and assessing their relative excellence. Work on the violin and the organ-pipe in particular was described.

Section VII : Lightweight Structures. C. W. Kosten pointed out the difficulties in building an insulating wall between two rooms without excessive weight. Flexural stiffness and resistance must be introduced to compensate for the light weight, and resonances must be distributed over the gamut. Both cavity walls and filled double-wall structures are employed.

After this the Congress divided into Sections, in each of which ten to a dozen papers were read, that of Section VI being enlivened by demonstrations on various musical instruments varying from bagpipes to just-tempered organs.

On two days visits to Hilversum Radio and Philips. Industries, Eindhoven, were made.

\section{EUROPEAN ASSOCIATION OF EXPLORATION GEOPHYSICISTS}

\section{PARIS MEETING}

$T$

HE fourth meeting (second annual meeting) of the European Association of Exploration Geophysicists was held in Paris during May 20-22, when about a hundred and fifty members were present, the president, A. van Weelden, being in the chair. Membership now stands at more than five hundred and fifty, a remarkable achievement since the Association's inauguration in December 1951. Affiliation with the Society of Exploration Geophysicists (U.S.A.) has recently been approved, and a paper entitled "The Significance of Diffraction in the Investigation of Faults"' ${ }_{1}$ by T. Krey, presented at the Association's meeting in London in May 1952 and published in the Journal of the Society of Exploration Geophysicists (before the Association's own journal, Geophysical Prospecting, was in being), has received the Society's award for the best paper during 1952 :

In his presidential address, A. van Weelden discussed the various ways in which the results of gravity surveys are analysed, and emphasized the imperfections and ambiguities in the interpretations which are brought about by unjustified assumptions. In this regard, he stressed the necessity for close co-operation between geologist and geophysicist, a necessity which is sometimes not given full support.

Of the twenty-two papers presented at the meeting, seven were on seismic, six on electrical, four on magnetic, two on gravity and three on general problems in exploration geophysics. 